\title{
ON THE ORBITAL STABILITY OF SOLITARY WAVES FOR THE 2-COUPLED NONLINEAR SCHRÖDINGER SYSTEM*
}

\author{
NGHIEM V. NGUYEN ${ }^{\dagger}$
}

Abstract. The coupled nonlinear Schrödinger system

$$
\left\{\begin{array}{c}
i u_{t}+u_{x x}+\left(a|u|^{2}+b|v|^{2}\right) u=0 \\
i v_{t}+v_{x x}+\left(b|u|^{2}+c|v|^{2}\right) v=0
\end{array}\right.
$$

where $u, v$ are complex valued functions of $(x, t) \in \mathbb{R}^{2}$, and $a, b, c \in \mathbb{R}$ was previously studied by Nguyen and Wang. In that work, it was shown that for this system of equations, the interplay between components of solutions in terms of the parameters $a, b, c$ plays an important role in both the existence and stability of solitary wave. In particular, it was proved that solitary wave solutions of this system are orbitally stable when either $0<b<\min \{a, c\}$, or $b>0$ with $b>\max \{a, c\}$ and $b^{2}>a c$. In this manuscript, the orbital stability result obtained by Nguyen and Wang is further improved. It will be shown that when a solitary wave is perturbed, the perturbed solution must stay close to a solitary-wave profile in which the translation and phase parameters are prescribed functions of time. Properties of these functions are then studied.

Key words. Coupled nonlinear Schrödinger equations, ground states, solitary-waves, orbital stability.

AMS subject classifications. 35A15, 35B35.

\section{Introduction}

The coupled nonlinear Schrödinger (CNLS) system

$$
\left\{\begin{array}{c}
i u_{t}+u_{x x}+\left(a|u|^{2}+b|v|^{2}\right) u=0 \\
i v_{t}+v_{x x}+\left(b|u|^{2}+c|v|^{2}\right) v=0
\end{array}\right.
$$

where $u, v$ are complex valued functions of $(x, t) \in \mathbb{R}^{2}$, and $a, b, c \in \mathbb{R}$, arises physically under conditions similar to those described by the well-understood cubic, nonlinear Schrödinger (NLS) equation

$$
i u_{t}+u_{x x} \pm|u|^{2} u=0
$$

For example, in optical fibers and waveguides, propagation of the electromagnetic waves is described by (1.1). When $a=b=c=1$, the CNLS system models physical systems in which there are two wavetrains moving with nearly the same group velocities. The CNLS system also arises in the Hartree-Fock theory for a double condensate, i.e., a binary mixture of Bose-Einstein condensates in two different hyperfine states. Readers are referred to the works [6, 12, 13, 24, 25] for the derivation as well as applications of this system.

The system (1.1) has the following conserved quantities:

$$
E(u, v)=\int_{\mathbb{R}}\left[\left|u_{x}(x, t)\right|^{2}+\left|v_{x}(x, t)\right|^{2}-\frac{a}{2}|u(x, t)|^{4}-\frac{c}{2}|v(x, t)|^{4}-b|u(x, t)|^{2}|v(x, t)|^{2}\right] d x,
$$

*Received: November 10, 2010; accepted (in revised version): February 22, 2011. Communicated by Jack Xin.

${ }^{\dagger}$ Department of Mathematics and Statistics, Utah State University, Logan, UT 84322, USA (nghiem.nguyen@usu.edu). 


$$
P(u)=\int_{-\infty}^{\infty}|u(x, t)|^{2} d x
$$

and

$$
P(v)=\int_{-\infty}^{\infty}|v(x, t)|^{2} d x
$$

In other words, when applied to sufficiently regular solutions $(u(x, t), v(x, t))$ of $(1.1)$, $E$ and $P$ are independent of $t$.

Solitary wave solutions of (1.1) of interest here have the form

$$
(u(x, t), v(x, t))=\left(e^{i\left[\left(\omega-\frac{1}{4} \theta^{2}\right) t+\left(\frac{1}{2} \theta x+m\right)\right]} A \varphi_{\omega}(x-\theta t), e^{i\left[\left(\omega-\frac{1}{4} \theta^{2}\right) t+\left(\frac{1}{2} \theta x+n\right)\right]} B \varphi_{\omega}(x-\theta t)\right)
$$

for $m, n$ real constants and $\omega, \theta \in \mathbb{R}$, with $\omega-\frac{1}{4} \theta^{2}>0$ and where $A=\sqrt{\frac{b-c}{b^{2}-a c}}>0$ and $B=\sqrt{\frac{b-a}{b^{2}-a c}}>0$. An important special case arises when $m=n=\theta=0$ and $\omega=\Omega>0$. These special solutions

$$
(u(x, t), v(x, t))=\left(e^{i \Omega t} A \phi_{\Omega}(x), e^{i \Omega t} B \phi_{\Omega}(x)\right),
$$

where

$$
\phi_{\Omega}(\xi)=\sqrt{2 \Omega} \operatorname{sech}(\sqrt{\Omega} \xi),
$$

are often referred to as standing waves. Notice that the function $\phi_{\Omega}$ must satisfy the equation

$$
-\frac{d^{2} \phi}{d x^{2}}+\Omega \phi-|\phi|^{2} \phi=0
$$

In [17] (see also [18]), it was shown that those standing waves are indeed ground states, that is, solutions that minimize energy $E$ subject to a fixed charge $P$. Moreover, these ground states are orbitally stable. In essence, the results obtained in [18] and [17] take the following form. Suppose $\left(e^{i \Omega t} f, e^{i \Omega t} g\right)$ is a ground-state solution of (1.1). Then for every $\epsilon>0$, there exists $\delta>0$ such that if $(u, v)$ is the solution of (1.1) with initial data $\left(u_{0}, v_{0}\right)$ that satisfies

$$
\inf _{\gamma_{1}, \gamma_{2}, y \in \mathbb{R}}\left\{\left\|u_{0}-e^{i \gamma_{1}} f(\cdot+y)\right\|_{H^{1}}+\left\|v_{0}-e^{i \gamma_{2}} g(\cdot+y)\right\|_{H^{1}}\right\}<\delta
$$

then

$$
\inf _{\theta_{1}, \theta_{2}, y \in \mathbb{R}}\left\{\left\|u(\cdot, t)-e^{i \theta_{1}} f(\cdot+y)\right\|_{H^{1}}+\left\|v(\cdot, t)-e^{i \theta_{2}} g(\cdot+y)\right\|_{H^{1}}\right\}<\epsilon
$$

for all $t \geq 0$. Define the orbit of the ground-state solutions to be the collection of all translations in the spatial variable, together with the two phase shifts $\left\{\left(e^{i \theta_{1}} f(x+\right.\right.$ $\left.\left.r), e^{i \theta_{2}} g(x+r)\right)\right\}_{r \in \mathbb{R}}$. Then the above result states that the ground-state solutions are orbitally stable.

While these results are attractive, they still leave open the question of the speed with which the solution $(u, v)$ propagates. For example, for the Korteweg-de Vries type equations it is known that the bulk of the disturbance flowing out of the perturbation 
travels at a speed near to the speed of the unperturbed solitary wave. Another interesting question is in regard to the phase with which the solution propagates. Because the system (1.1) is invariant under the Galilean transformation

$$
(u(x, t), v(x, t)) \rightarrow\left(e^{-i \frac{1}{4} \theta^{2} t+i \frac{1}{2} \theta x} u(x-\theta t, t), e^{-i \frac{1}{4} \theta^{2} t+i \frac{1}{2} \theta x} v(x-\theta t, t)\right), \quad \theta \in \mathbb{R},
$$

and the phase transformation

$$
(u(x, t), v(x, t)) \rightarrow\left(e^{i m} u(x, t), e^{i n} v(x, t)\right), \quad m, n \in \mathbb{R},
$$

one can always obtain the solitary-wave solutions as in (1.6) from ground states, but then it is not clear how the phase shifts will be affected in the previously stated stability results.

In this manuscript, these questions will be addressed. It will be shown first that when a ground state is perturbed, the perturbed solution must stay close to a ground state profile in which the translation and phase parameters are prescribed $C^{1}$-functions of time. The precise statement for this first part is as follows.

THEOREM 1.1. The ground-state solution $\left(e^{i \Omega t} A \phi_{\Omega}, e^{i \Omega t} B \phi_{\Omega}\right)$ is orbitally stable in the sense that for any $\epsilon>0$, there exists $\delta=\delta(\epsilon)>0$ such that if

$$
\inf _{\gamma_{1}, \gamma_{2}, y \in \mathbb{R}}\left\{\left\|u_{0}-e^{i \gamma_{1}} A \phi_{\Omega}(\cdot+y)\right\|_{H^{1}}+\left\|v_{0}-e^{i \gamma_{2}} B \phi_{\Omega}(\cdot+y)\right\|_{H^{1}}\right\}<\delta
$$

then there are $C^{1}$-mappings $\theta_{1}, \theta_{2}, \eta: \mathbb{R} \rightarrow \mathbb{R}$ for which the solution $(u(x, t), v(x, t))$ emanating from the initial data $(u(\cdot, 0), v(\cdot, 0))=\left(u_{0}, v_{0}\right)$ satisfies

$$
\left\|u(\cdot, t)-e^{i \theta_{1}(t)} A \phi_{\Omega}(\cdot-\eta(t))\right\|_{H^{1}}+\left\|v(\cdot, t)-e^{i \theta_{2}(t)} B \phi_{\Omega}(\cdot-\eta(t))\right\|_{H^{1}}<\epsilon
$$

for all $t \geq 0$. Moreover,

$$
\begin{aligned}
& \eta^{\prime}(t)=O(\epsilon), \\
& \theta_{i}^{\prime}(t)=\Omega+O(\epsilon)
\end{aligned}
$$

for $i=1,2$ as $\epsilon \rightarrow 0$, uniformly in $t$.

The central argument is based on the fact that, following the ideas introduced in $[8,23]$, the functions $\eta$ and $\theta_{i}$ are chosen to satisfy the following orthogonality relations:

$$
\begin{aligned}
& \operatorname{Re}\left\{\int_{-\infty}^{\infty} A \phi_{\Omega}^{2}(x) \phi_{\Omega}^{\prime}(x)\left(e^{-i \theta_{1}(t)} u(x+\eta(t), t)\right) d x\right. \\
& \left.\quad+\int_{-\infty}^{\infty} B \phi_{\Omega}^{2}(x) \phi_{\Omega}^{\prime}(x)\left(e^{-i \theta_{2}(t)} v(x+\eta(t), t)\right) d x\right\}=0 ; \\
& \mathcal{I} m\left\{\int_{-\infty}^{\infty} A \phi_{\Omega}^{3}(x)\left(e^{-i \theta_{1}(t)} u(x+\eta(t), t)\right) d x\right\}=0 ; \\
& \mathcal{I} m\left\{\int_{-\infty}^{\infty} B \phi_{\Omega}^{3}(x)\left(e^{-i \theta_{2}(t)} v(x+\eta(t), t)\right) d x\right\}=0 .
\end{aligned}
$$

Indeed, (1.10) results from the first-order conditions corresponding to minimizing the function $R\left(\theta_{1}, \theta_{2}, \eta\right)$ defined as

$$
\begin{aligned}
R\left(\theta_{1}, \theta_{2}, \eta\right)= & \Omega\left\|e^{-i \theta_{1}} u(\cdot+\eta)-A \phi_{\Omega}\right\|_{L^{2}}^{2}+\Omega\left\|e^{-i \theta_{2}} v(\cdot+\eta)-B \phi_{\Omega}\right\|_{L^{2}}^{2} \\
& +\left\|e^{-i \theta_{1}} u^{\prime}(\cdot+\eta)-A \phi_{\Omega}^{\prime}\right\|_{L^{2}}^{2}+\left\|e^{-i \theta_{2}} v^{\prime}(\cdot+\eta)-B \phi_{\Omega}^{\prime}\right\|_{L^{2}}^{2}
\end{aligned}
$$


and Equation (2.5) below, which is satisfied by the ground state.

Next, the result is extended to include solitary-wave solutions as well. The argument for this part is based on the following fact. For any pair $(\omega, \theta) \in \mathbb{R} \times \mathbb{R}$ such that $\Omega=\omega-\frac{1}{4} \theta^{2}>0$, let $\varphi_{\omega}=T_{\theta} \phi_{\Omega}$, where the operator $T_{\theta}: H_{\mathbb{C}}^{1}(\mathbb{R}) \rightarrow H_{\mathbb{C}}^{1}(\mathbb{R})$ is defined by

$$
\left(T_{\theta} u\right)(x)=\exp \left(\frac{i \theta x}{2}\right) u(x)
$$

for $u \in H_{\mathbb{C}}^{1}(\mathbb{R})$. Then if $\left(e^{i \Omega t} A \phi_{\Omega}, e^{i \Omega t} B \phi_{\Omega}\right)$ is a ground state of (1.1), then $\left(e^{i \omega t} A \varphi_{\omega}, e^{i \omega t} B \varphi_{\omega}\right)$ is a solitary-wave solution of (1.1).

It will be shown further that the solution emanating from a perturbed solitary wave travels at nearly the same speed and phase shifts as the unperturbed solitary wave. Such a result is to be expected since a similar one has been established for the cubic, nonlinear Schrödinger Equation (1.2), an equation that arises physically under conditions similar to those described by (1.1) (see, for example, $[8,9,23]$ ). The precise statement for this last part is as follows.

THEOREM 1.2. The solitary-wave solutions ( $\left(e^{i \omega t} A \varphi_{\omega}, e^{i \omega t} B \varphi_{\omega}\right)$ of (1.1) are orbitally stable in the sense that for any $\epsilon>0$ given, there exists $\delta=\delta(\epsilon)>0$ such that if

$$
\inf _{\gamma_{1}, \gamma_{2}, y \in \mathbb{R}}\left\{\left\|u_{0}-e^{i \gamma_{1}} A \varphi_{\omega}(\cdot+y)\right\|_{H^{1}}+\left\|v_{0}-e^{i \gamma_{2}} B \varphi_{\omega}(\cdot+y)\right\|_{H^{1}}\right\} \leq \delta
$$

then there are $C^{1}$-mappings $p_{1}, p_{2}, q: \mathbb{R} \rightarrow \mathbb{R}$ for which the solution $(u, v)$ of (1.1) emanating from the initial data $\left(u_{0}, v_{0}\right)$ satisfies

$$
\left\|u(\cdot, t)-e^{i p_{1}(t)} A \varphi_{\omega}(\cdot-q(t))\right\|_{H^{1}}+\left\|v(\cdot, t)-e^{i p_{2}(t)} B \varphi_{\omega}(\cdot-q(t))\right\|_{H^{1}} \leq \epsilon
$$

for all $t \geq 0$. Moreover, $p_{1}, p_{2}$ and $q$ are close to $\omega$ and $\theta$ in the sense that

$$
\begin{aligned}
p_{1}^{\prime}(t) & =\omega+O(\epsilon), \\
p_{2}^{\prime}(t) & =\omega+O(\epsilon), \\
q^{\prime}(t) & =\theta+O(\epsilon)
\end{aligned}
$$

as $\epsilon \rightarrow 0$, uniformly in $t$.

Notation: For $1 \leq p \leq \infty$, we denote by $L^{p}=L^{p}(\mathbb{R})$ the space of all measurable functions $f$ on $\mathbb{R}$ for which the norm $|f|_{p}=\left(\int_{-\infty}^{\infty}|f|^{p} d x\right)^{1 / p}$ is finite for $1 \leq p<\infty$ and $|f|_{\infty}$ is the essential supremum of $|f|$ on $\mathbb{R}$. Whether we intend the functions in $L^{p}$ to be real-valued or complex-valued will be clear from the context. $H_{\mathbb{C}}^{1}(\mathbb{R})$ is the usual Sobolev space consisting of measurable, complex-valued functions such that both $f$ and $f_{x}$ are in $L^{2}$. Let $X$ be a Banach space defined by the Cartesian product $H_{\mathbb{C}}^{1}(\mathbb{R}) \times H_{\mathbb{C}}^{1}(\mathbb{R})$. If $T>0$ and $Y$ is any Banach space, we denote by $C([0, T], Y)$ the Banach space of continuous maps $f:[0, T] \rightarrow Y$, with norms given by $\|f\|_{C([0, T], Y)}=\sup _{t \in[0, T]}\|f(t)\|_{Y}$.

This manuscript is organized as follows. In Section 2, a review of existing theory for the 2-coupled nonlinear Schrödinger system (1.1) is presented, along with a summary of the main contribution of this manuscript. In Section 3, it will be shown that the orbital stability result obtained in [17] for ground states can be improved. The improvement is made by picking unique trajectory and phase shifts that the perturbed ground states must follow. Properties of these maps are also studied in detail. These results are then extended in Section 4 to include solitary-wave solutions as well. 


\section{Review of existing theory}

In the case when $a=c>-1, b=1$ (also known as the symmetric case), the nonlinear Schrödinger system (1.1) is known to have explicit solitary wave solution of the form (see, for example, [18])

$$
\left(u_{\omega}(x, t), v_{\omega}(x, t)\right)=\left(e^{i\left(\omega-\frac{1}{4} \theta^{2}\right) t+i \frac{1}{2} \theta x+i m} \tilde{\varphi}_{\omega}(x-\theta t), e^{i\left(\omega-\frac{1}{4} \theta^{2}\right) t+i \frac{1}{2} \theta x+i n} \tilde{\varphi}_{\omega}(x-\theta t)\right)
$$

for $m, n$ real constants and $\omega, \theta \in \mathbb{R}$ with $\omega-\frac{1}{4} \theta^{2}>0$ and

$$
\tilde{\varphi}_{\omega}(x-\theta t)=\sqrt{\frac{2 \omega}{a+1}} \operatorname{sech}(\sqrt{\omega}(x-\theta t))
$$

Since the system (1.1) is invariant under the Galilean and phase transformations, one may consider the case when $\theta=m=n=0$ and $\omega=\Omega>0$ in (2.1) to obtain the following standing waves:

$$
\left(u_{\Omega}(x, t), v_{\Omega}(x, t)\right)=\left(e^{i \Omega t} \sqrt{\frac{1}{a+1}} \phi_{\Omega}(x), e^{i \Omega t} \sqrt{\frac{1}{a+1}} \phi_{\Omega}(x)\right)
$$

where $\phi_{\Omega}(x)$ is given as in (1.8).

It has been proved by Ohta [18] that in fact these standing waves are ground states; moreover, these ground state solutions are stable. In particular, his result is as follows:

Let $a=c>-1$ and $b=1$. For any $\Omega>0$, the ground states $\left(u_{\Omega}(x, t), v_{\Omega}(x, t)\right)$ are orbitally stable in the following sense: for any $\epsilon>0$, there exists $\delta>0$ such that if $\left(u_{0}, v_{0}\right) \in X$ satisfies

$$
\inf _{\gamma_{1}, \gamma_{2}, y \in \mathbb{R}}\left\{\left\|u_{0}-\sqrt{\frac{1}{a+1}} e^{i \gamma_{1}} \phi_{\Omega}(\cdot+y)\right\|_{H^{1}}+\left\|v_{0}-\sqrt{\frac{1}{a+1}} e^{i \gamma_{2}} \phi_{\Omega}(\cdot+y)\right\|_{H^{1}}\right\}<\delta,
$$

then the solution $(u(x, t), v(x, t))$ with $(u(\cdot, 0), v(\cdot, 0))=\left(u_{0}, v_{0}\right)$ satisfies

$$
\begin{gathered}
\sup _{t \in \mathbb{R}} \inf _{\theta_{1}, \theta_{2}, y \in \mathbb{R}}\left\{\left\|u(\cdot, t)-e^{i \theta_{1}} \sqrt{\frac{1}{a+1}} \phi_{\Omega}(\cdot+y)\right\|_{H^{1}}\right. \\
\left.+\left\|v(\cdot, t)-e^{i \theta_{2}} \sqrt{\frac{1}{a+1}} \phi_{\Omega}(\cdot+y)\right\|_{H^{1}}\right\}<\epsilon .
\end{gathered}
$$

Ohta's result was extended to include more general settings in [17], namely, the nonsymmetric case $a \neq c$. Precisely, it was assumed that either

(A1) $0<b<\min \{a, c\}$;

or

(A2) $b>0$ with $b>\max \{a, c\}$ and $b^{2}>a c$. 
For these values of $a, b$, and $c$ and for some fixed $\Omega>0$, consider the standing waves

$$
\left(e^{i \Omega t} \sqrt{\frac{b-c}{b^{2}-a c}} \phi_{\Omega}(x), e^{i \Omega t} \sqrt{\frac{b-a}{b^{2}-a c}} \phi_{\Omega}(x)\right)
$$

where $\phi_{\Omega}$ is as given in (1.8). These standing waves are indeed ground states and they are stable. The precise results (see [17]) are as follows.

THEOREM 2.1. Let $a, b$, and $c$ be real numbers such that either (A1) or (A2) is satisfied. Then, for any $\Omega>0$ the ground-state solutions given by (2.4)-(1.8) are orbitally stable in the following sense: for any $\epsilon>0$, there exists $\delta>0$ such that if $\left(u_{0}, v_{0}\right) \in X$ satisfies

$$
\inf _{\gamma_{1}, \gamma_{2}, y \in \mathbb{R}}\left\{\left\|u_{0}-\sqrt{\frac{b-c}{b^{2}-a c}} e^{i \gamma_{1}} \phi_{\Omega}(\cdot+y)\right\|_{H^{1}}+\left\|v_{0}-\sqrt{\frac{b-a}{b^{2}-a c}} e^{i \gamma_{2}} \phi_{\Omega}(\cdot+y)\right\|_{H^{1}}\right\}<\delta
$$

then the solution $(u(x, t), v(x, t))$ with $(u(\cdot, 0), v(\cdot, 0))=\left(u_{0}, v_{0}\right)$ satisfies

$$
\inf _{\theta_{1}, \theta_{2}, y \in \mathbb{R}}\left\{\left\|u-\sqrt{\frac{b-c}{b^{2}-a c}} e^{i \theta_{1}} \phi_{\Omega}(\cdot+y)\right\|_{H^{1}}+\left\|v-\sqrt{\frac{b-a}{b^{2}-a c}} e^{i \theta_{2}} \phi_{\Omega}(\cdot+y)\right\|_{H^{1}}\right\}<\epsilon
$$

uniformly for all $t \geq 0$.

In the next section, it will be shown that instead of allowing the ground-state solutions to wander around at random, one can pick unique trajectory and phase shifts that the ground-state solutions must follow. Properties of these maps are then studied.

REMARK 2.2. 1) In the last several years there have been intensive works studying the existence of standing waves for nonlinear Schrödinger systems of the form studied in this paper; for example, see $[1,2,3,4,5,11,14,15,19]$ and references therein. Most of these papers are concerned with the corresponding nonlinear elliptic systems and various methods have been employed to construct solutions for various parameter regimes.

2) In [10] a different variational setting than the one in [17] is used to prove the stability of solitary waves for (1.1), namely using the sum of the $L^{2}$-norms of the two components. The two variational problems can have different solitary-wave solutions. In fact, the last two pages of [10] show that in the case when $a=c=\alpha$ and $b<\alpha$, the solitary waves which solve the variational problem in [17] are not the same as the solitary waves which solve the variational problem in [10].

3) In [20, 21], Song proves stability of standing waves to a system of Schrödinger equations with combined power-type nonlinearities, which includes (1.1) when the dimension is $n=1$. However, due to the nature of problem being posed in higher dimensions, uniqueness of the ground-state solutions is not studied in [20, 21]; moreover, the range of stability for the coefficients $a, b, c$ is smaller compared to the one obtained in [17] (namely, in [20] $a, b, c$ are strictly positive while $a, c$ are allowed to be negative as well in [17]).

4) Notice that for any fixed $\Omega>0$, the ground states satisfy

$$
\left\{\begin{array}{l}
-A \phi_{\Omega}^{\prime \prime}+\Omega A \phi_{\Omega}=\left(a\left|A \phi_{\Omega}\right|^{2}+b\left|B \phi_{\Omega}\right|^{2}\right) A \phi_{\Omega}, \\
-B \phi_{\Omega}^{\prime \prime}+\Omega B \phi_{\Omega}=\left(b\left|A \phi_{\Omega}\right|^{2}+c\left|B \phi_{\Omega}\right|^{2}\right) B \phi_{\Omega}
\end{array}\right.
$$


where $A=\sqrt{\frac{b-c}{b^{2}-a c}}>0$ and $B=\sqrt{\frac{b-a}{b^{2}-a c}}>0$. In [22], Wei and Yao have established uniqueness of positive solutions to (2.5) for a range of $a, b$, and $c$. They proved that for $0 \leq b \notin\{\min (a, c), \max (a, c)\}$, all solutions are given by $\left(A \phi_{\Omega}, B \phi_{\Omega}\right)$. In fact, when $b>0$ all possible solitary waves are classified and the nondegeneracy is also proven in [22].

\section{Result for ground-state solutions}

As noted in $[8,23]$, the crucial argument for choosing the functions $\theta_{1}, \theta_{2}, \eta$ is deduced from appreciating that the first-order conditions corresponding to minimizing the function

$$
\begin{aligned}
R\left(\theta_{1}, \theta_{2}, \eta\right)= & \Omega\left\|e^{-i \theta_{1}} u(\cdot+\eta)-A \phi_{\Omega}\right\|_{L^{2}}^{2}+\Omega\left\|e^{-i \theta_{2}} v(\cdot+\eta)-B \phi_{\Omega}\right\|_{L^{2}}^{2} \\
& +\left\|e^{-i \theta_{1}} u^{\prime}(\cdot+\eta)-A \phi_{\Omega}^{\prime}\right\|_{L^{2}}^{2}+\left\|e^{-i \theta_{2}} v^{\prime}(\cdot+\eta)-B \phi_{\Omega}^{\prime}\right\|_{L^{2}}^{2}
\end{aligned}
$$

produce the orthogonality relations

$$
\begin{aligned}
& \operatorname{Re}\left\{\int_{-\infty}^{\infty} A \phi_{\Omega}^{2}(x) \phi_{\Omega}^{\prime}(x)\left(e^{-i \theta_{1}} u(x+\eta, t)\right) d x\right. \\
& \left.\quad+\int_{-\infty}^{\infty} B \phi_{\Omega}^{2}(x) \phi_{\Omega}^{\prime}(x)\left(e^{-i \theta_{2}} v(x+\eta, t)\right) d x\right\}=0 ; \\
& \operatorname{Im}\left\{\int_{-\infty}^{\infty} A \phi_{\Omega}^{3}(x)\left(e^{-i \theta_{1}} u(x+\eta, t)\right) d x\right\}=0 ; \\
& \mathcal{I} m\left\{\int_{-\infty}^{\infty} B \phi_{\Omega}^{3}(x)\left(e^{-i \theta_{2}} v(x+\eta, t)\right) d x\right\}=0 .
\end{aligned}
$$

Following this idea, we consider the vector-valued function $Q: X \times \mathbb{R}^{3} \longrightarrow \mathbb{R}^{3}$ defined by

$$
Q\left(\left(\psi_{1}, \psi_{2}\right), \theta_{1}, \theta_{2}, \eta\right)=(F, G, H)
$$

where

$$
\begin{aligned}
& F\left(\left(\psi_{1}, \psi_{2}\right), \theta_{1}, \theta_{2}, \eta\right)=\mathcal{R} e\left\{\int_{-\infty}^{\infty} A \phi_{\Omega}^{2}(x) \phi_{\Omega}^{\prime}(x)\left(e^{-i \theta_{1}} \psi_{1}(x+\eta)\right) d x\right. \\
& \left.+\int_{-\infty}^{\infty} B \phi_{\Omega}^{2}(x) \phi_{\Omega}^{\prime}(x)\left(e^{-i \theta_{2}} \psi_{2}(x+\eta)\right) d x\right\} ; \\
& G\left(\left(\psi_{1}, \psi_{2}\right), \theta_{1}, \theta_{2}, \eta\right)=\mathcal{I} m\left\{\int_{-\infty}^{\infty} A \phi_{\Omega}^{3}(x)\left(e^{-i \theta_{1}} \psi_{1}(x+\eta)\right) d x\right\} ; \\
& H\left(\left(\psi_{1}, \psi_{2}\right), \theta_{1}, \theta_{2}, \eta\right)=\mathcal{I} m\left\{\int_{-\infty}^{\infty} B \phi_{\Omega}^{3}(x)\left(e^{-i \theta_{2}} \psi_{2}(x+\eta)\right) d x\right\} .
\end{aligned}
$$

The following Lemma is needed in defining the advertised $C^{1}$-maps.

Lemma 3.1. When evaluating at $\left(\left(A \phi_{\Omega}, B \phi_{\Omega}\right), 0,0,0\right)$, one has
a) $Q\left(\left(A \phi_{\Omega}, B \phi_{\Omega}\right), 0,0,0\right)=(0,0,0)$;
b) $\frac{\partial Q}{\partial\left(\theta_{1}, \theta_{2}, \eta\right)}\left(\left(A \phi_{\Omega}, B \phi_{\Omega}\right), 0,0,0\right)<0$. 
Proof. Statement $a)$ follows easily from the fact that $\phi_{\Omega}(x)=\sqrt{2 \Omega} \operatorname{sech}(\sqrt{\Omega} x)$ and

$$
\int_{-\infty}^{\infty} \operatorname{sech}^{4} x d x=\frac{4}{3}
$$

Another calculation reveals that at the point $\left(\left(A \phi_{\Omega}, B \phi_{\Omega}\right), 0,0,0\right)$, the value for $\frac{\partial Q}{\partial\left(\theta_{1}, \theta_{2}, \eta\right)}$ is

$$
\begin{gathered}
\operatorname{det}\left[\begin{array}{ccc}
0 & 0 & -\left(A^{2}+B^{2}\right) \int_{-\infty}^{\infty} \phi_{\Omega}^{2}(x)\left(\phi_{\Omega}^{\prime}(x)\right)^{2} d x \\
-A^{2} \int_{-\infty}^{\infty} \phi_{\Omega}^{4}(x) d x & 0 & 0 \\
0 & -B^{2} \int_{-\infty}^{\infty} \phi_{\Omega}^{4}(x) d x & 0
\end{array}\right] \\
=-A^{2} B^{2}\left(A^{2}+B^{2}\right)\left(\int_{-\infty}^{\infty} \phi_{\Omega}^{4}(x) d x\right)^{2}\left(\int_{-\infty}^{\infty} \phi_{\Omega}^{2}(x)\left(\phi_{\Omega}^{\prime}(x)\right)^{2} d x\right)<0,
\end{gathered}
$$

which proves statement $b$ ).

For $\beta>0$, define an $X$-neighborhood of the trajectory of $\left(e^{i \theta_{1}} A \phi_{\Omega}, e^{i \theta_{2}} B \phi_{\Omega}\right)$ by

$$
\begin{gathered}
\mathcal{U}_{\beta}=\left\{\left(\psi_{1}, \psi_{2}\right) \in X: \inf _{\theta_{1}, \theta_{2}, \eta \in \mathbb{R}}\left\{\left\|e^{-i \theta_{1}} \psi_{1}(\cdot+\eta)-A \phi_{\Omega}\right\|_{H^{1}}\right.\right. \\
\left.\left.+\left\|e^{-i \theta_{2}} \psi_{2}(\cdot+\eta)-B \phi_{\Omega}\right\|_{H^{1}}\right\}<\beta\right\} .
\end{gathered}
$$

The next Lemma provides a choice of functions $\theta_{1}, \theta_{2}, \eta$ by demanding the satisfaction of the orthogonality condition (3.3).

Lemma 3.2. Fix $\Omega>0$. There exist $\beta>0$ and $C^{1}$-maps

$$
\theta_{1}, \theta_{2}, \eta: \mathcal{U}_{\beta} \longrightarrow \mathbb{R}
$$

such that for all $\left(\psi_{1}, \psi_{2}\right) \in \mathcal{U}_{\beta}$ the following statements hold:

1) $\mathcal{R} e\left\{\int_{-\infty}^{\infty} A \phi_{\Omega}^{2}(x) \phi_{\Omega}^{\prime}(x)\left(e^{-i \theta_{1}\left(\psi_{1}, \psi_{2}\right)} \psi_{1}\left(x+\eta\left(\psi_{1}, \psi_{2}\right)\right)\right) d x\right.$

$$
\left.+\int_{-\infty}^{\infty} B \phi_{\Omega}^{2}(x) \phi_{\Omega}^{\prime}(x)\left(e^{-i \theta_{2}\left(\psi_{1}, \psi_{2}\right)} \psi_{2}\left(x+\eta\left(\psi_{1}, \psi_{2}\right)\right)\right) d x\right\}=0
$$

2) $\operatorname{Im}\left\{\int_{-\infty}^{\infty} A \phi_{\Omega}^{3}(x)\left(e^{-i \theta_{1}\left(\psi_{1}, \psi_{2}\right)} \psi_{1}\left(x+\eta\left(\psi_{1}, \psi_{2}\right)\right)\right) d x\right\}=0$;

3) $\operatorname{I} m\left\{\int_{-\infty}^{\infty} B \phi_{\Omega}^{3}(x)\left(e^{-i \theta_{2}\left(\psi_{1}, \psi_{2}\right)} \psi_{2}\left(x+\eta\left(\psi_{1}, \psi_{2}\right)\right)\right) d x\right\}=0$,

with $\theta_{i}\left(A \phi_{\Omega}, B \phi_{\Omega}\right)=0$ for $i=1,2, \eta\left(A \phi_{\Omega}, B \phi_{\Omega}\right)=0$, and

$$
Q\left(\left(\psi_{1}, \psi_{2}\right), \theta_{1}\left(\psi_{1}, \psi_{2}\right), \theta_{2}\left(\psi_{1}, \psi_{2}\right), \eta\left(\psi_{1}, \psi_{2}\right)\right)=(0,0,0) .
$$

Moreover,

$$
\eta\left(\psi_{1}(\cdot+\tau), \psi_{2}(\cdot+\tau)\right)=\eta\left(\psi_{1}, \psi_{2}\right)+\tau
$$


Proof. It is clear that $Q$ is $C^{1}$ on $\mathcal{U} \times \mathbb{R}^{3}$. In fact, the derivatives of $Q$ with respect to components $\eta$ and $\theta_{i}$ exist up to any order. Now, for any $\rho>0$, denote the ball centered at $\left(e^{i \theta_{1}} A \phi_{\Omega}, e^{\theta_{2}} B \phi_{\Omega}\right) \in X$ with radius $\rho$ as

$$
\begin{aligned}
& \mathcal{B}_{\rho}\left(e^{i \theta_{1}} A \phi_{\Omega}, e^{i \theta_{2}} B \phi_{\Omega}\right) \\
= & \left\{\left(\psi_{1}, \psi_{2}\right) \in X:\left\|e^{-i \theta_{1}} \psi_{1}-A \phi_{\Omega}\right\|_{H^{1}}+\left\|e^{-i \theta_{2}} \psi_{2}-B \phi_{\Omega}\right\|_{H^{1}}<\rho\right\} .
\end{aligned}
$$

The implicit function theorem together with Lemma 3.1 imply the existence of positive numbers $\beta, r$ and unique $C^{1}$-functions

$$
\theta_{1}, \theta_{2}, \eta: \quad \mathcal{B}_{\beta}\left(e^{i \theta_{1}} A \phi_{\Omega}, e^{i \theta_{2}} B \phi_{\Omega}\right) \longrightarrow(-r, r)
$$

such that for all $\left(\psi_{1}, \psi_{2}\right) \in \mathcal{B}_{\beta}\left(e^{i \theta_{1}} A \phi_{\Omega}, e^{i \theta_{2}} B \phi_{\Omega}\right)$,

$$
\begin{aligned}
& \theta_{i}\left(A \phi_{\Omega}, B \phi_{\Omega}\right)=0, \quad i=1,2 ; \\
& \eta\left(A \phi_{\Omega}, B \phi_{\Omega}\right)=0 ; \quad \text { and } \\
& Q\left(\left(\psi_{1}, \psi_{2}\right), \theta_{1}\left(\psi_{1}, \psi_{2}\right), \theta_{2}\left(\psi_{1}, \psi_{2}\right), \eta\left(\psi_{1}, \psi_{2}\right)\right)=(0,0,0) .
\end{aligned}
$$

The first three statements now follow. To see that $\eta\left(\psi_{1}(\cdot+\tau), \psi_{2}(\cdot+\tau)\right)=\eta\left(\psi_{1}, \psi_{2}\right)+$ $\tau$, let $\left(\psi_{1}, \psi_{2}\right) \in \mathcal{B}_{\beta}\left(e^{i \theta_{1}} A \phi_{\Omega}, e^{i \theta_{2}} B \phi_{\Omega}\right)$ and $\tau \in \mathbb{R}$ be such that $\left(\psi_{1}(\cdot+\tau), \psi_{2}(\cdot+\tau)\right) \in$ $\mathcal{B}_{\beta}\left(e^{i \theta_{1}} A \phi_{\Omega}, e^{i \theta_{2}} B \phi_{\Omega}\right)$. Then the translation invariance of Lebesgue measure says that

1) $\mathcal{R} e\left\{\int_{-\infty}^{\infty} A \phi_{\Omega}^{2}(x+\tau) \phi_{\Omega}^{\prime}(x+\tau)\left(e^{-i \theta_{1}\left(\psi_{1}, \psi_{2}\right)} \psi_{1}\left(x+\eta\left(\psi_{1}, \psi_{2}\right)+\tau\right)\right) d x\right.$

$$
\left.+\int_{-\infty}^{\infty} B \phi_{\Omega}^{2}(x+\tau) \phi_{\Omega}^{\prime}(x+\tau)\left(e^{-i \theta_{2}\left(\psi_{1}, \psi_{2}\right)} \psi_{2}\left(x+\eta\left(\psi_{1}, \psi_{2}\right)+\tau\right)\right) d x\right\}=0 ;
$$

2) $\mathcal{I} m\left\{\int_{-\infty}^{\infty} A \phi_{\Omega}^{3}(x+\tau)\left(e^{-i \theta_{1}\left(\psi_{1}, \psi_{2}\right)} \psi_{1}\left(x+\eta\left(\psi_{1}, \psi_{2}\right)+\tau\right)\right) d x\right\}=0$;

3) $\operatorname{I} m\left\{\int_{-\infty}^{\infty} B \phi_{\Omega}^{3}(x+\tau)\left(e^{-i \theta_{2}\left(\psi_{1}, \psi_{2}\right)} \psi_{2}\left(x+\eta\left(\psi_{1}, \psi_{2}\right)+\tau\right)\right) d x\right\}=0$.

As the value of $\eta\left(\psi_{1}, \psi_{2}\right)$ is unique, it must be the case that

$$
\eta\left(\psi_{1}(\cdot+\tau), \psi_{2}(\cdot+\tau)\right)=\eta\left(\psi_{1}, \psi_{2}\right)+\tau .
$$

The mapping $\eta$ can be easily extended to all of $\mathcal{U}_{\beta}$, where $\beta$ is the radius assured by the implicit-function theorem. If for some $\tau \in \mathbb{R}$,

$$
\left\|e^{-i \theta_{1}} \psi_{1}(\cdot+\tau)-A \phi_{\Omega}\right\|_{H^{1}}+\left\|e^{-i \theta_{2}} \psi_{2}(\cdot+\tau)-B \phi_{\Omega}\right\|_{H^{1}}<\beta,
$$

then define $\eta\left(\psi_{1}, \psi_{2}\right)=\eta\left(\left(\psi_{1}(\cdot-\tau), \psi_{2}(\cdot-\tau)\right)\right)+\tau$. This definition makes sense since if

$$
\left\|e^{-i \theta_{1}} \psi_{1}\left(\cdot+\tau_{1}\right)-A \phi_{\Omega}\right\|_{H^{1}}+\left\|e^{-i \theta_{2}} \psi_{2}\left(\cdot+\tau_{1}\right)-B \phi_{\Omega}\right\|_{H^{1}}<\beta,
$$

then both $\left(\psi_{1}(\cdot-\tau), \psi_{2}(\cdot-\tau)\right)$ and $\left(\psi_{1}\left(\cdot-\tau_{1}\right), \psi_{2}\left(\cdot-\tau_{1}\right)\right)$ belong to $\mathcal{B}_{\beta}\left(e^{i \theta_{1}} A \phi_{\Omega}, e^{i \theta_{2}} B \phi_{\Omega}\right)$. Since (3.6) holds in $\mathcal{B}_{\beta}\left(e^{i \theta_{1}} A \phi_{\Omega}, e^{i \theta_{2}} B \phi_{\Omega}\right)$, it follows that

$$
\begin{aligned}
\eta\left(\psi_{1}\left(\cdot-\tau_{1}\right), \psi_{2}\left(\cdot-\tau_{1}\right)\right) & =\eta\left(\psi_{1}\left(\cdot-\tau-\left(\tau_{1}-\tau\right)\right), \psi_{2}\left(\cdot-\tau-\left(\tau_{1}-\tau\right)\right)\right) \\
& =\eta\left(\psi_{1}(\cdot-\tau), \psi_{2}(\cdot-\tau)\right)-\tau_{1}+\tau
\end{aligned}
$$


which is the same as

$$
\eta\left(\psi_{1}\left(\cdot-\tau_{1}\right), \psi_{2}\left(\cdot-\tau_{1}\right)\right)+\tau_{1}=\eta\left(\psi_{1}(\cdot-\tau), \psi_{2}(\cdot-\tau)\right)+\tau
$$

This completes the proof of Lemma 3.2.

REMARK 3.3. It follows immediately from Lemma 3.2 and the definition of the extension of $\eta$ to all of $\mathcal{U}_{\beta}$ that, for any $\epsilon$ with $0<\epsilon<r$, there exists a $\delta$ with $0<\delta<\beta$ such that for all $\left(\psi_{1}, \psi_{2}\right) \in \mathcal{B}_{\delta}\left(e^{i \theta_{1}} A \phi_{\Omega}(\cdot+\tau), e^{i \theta_{2}} B \phi_{\Omega}(\cdot+\tau)\right)$,

$$
\left|\eta\left(\psi_{1}, \psi_{2}\right)-\tau\right|<\epsilon
$$

Because of the stability result stated in Theorem 2.1, $(u(\cdot, t), v(\cdot, t)) \in \mathcal{U}_{\beta}$ and hence the corresponding functions $\theta_{1}, \theta_{2}$, and $\eta$ are defined on $(u(\cdot, t), v(\cdot, t))$; one can now consider the functions $\theta_{1}, \theta_{2}$, and $\eta$ from $\mathbb{R}$ to $\mathbb{R}$ as

$$
\eta(t)=\eta(u(\cdot, t), v(\cdot, t))
$$

and for $i=1,2$,

$$
\theta_{i}(t)=\theta_{i}(u(\cdot, t), v(\cdot, t))
$$

Notice that since $(u, v)$ is a solution of (1.1), it must satisfy

$$
\left\{\begin{array}{l}
u_{t}=i\left[u_{x x}+\left(a|u|^{2}+b|v|^{2}\right) u\right] \\
v_{t}=i\left[v_{x x}+\left(b|u|^{2}+c|v|^{2}\right) v\right]
\end{array}\right.
$$

Proposition 3.4. The function $Q$ as defined in (3.3) is continuously differentiable with respect to $t$.

Proof. It has been proved (Chapter 4 in [9]) that for any $(u(x, 0), v(x, 0)) \in X$, there exists a unique solution $(u(x, t), v(x, t))$ of $(1.1)$ in $C(\mathbb{R} ; X)$ emanating from $(u(x, 0), v(x, 0))$, and such that $(u(x, t), v(x, t))$ satisfies

$$
\begin{gathered}
P(u(x, t))=P(u(x, 0)), \quad P(v(x, t))=P(v(x, 0)), \\
E(u(x, t), v(x, t))=E(u(x, 0), v(x, 0)) .
\end{gathered}
$$

Thus $(u(x, t), v(x, t))$ is differentiable as a distribution-valued function of $t$ with $\left(u_{t}, v_{t}\right) \in C\left(\mathbb{R}, H^{-1} \times H^{-1}\right)$. Hence for any functions $\chi_{1}, \chi_{2}$ in the Schwarz class $\mathcal{S}$, the action

$$
\left\langle(u, v),\left(\chi_{1}, \chi_{2}\right)\right\rangle=\int_{-\infty}^{\infty}\left[u(x, t) \chi_{1}(x)+v(x, t) \chi_{2}(x)\right] d x
$$

of $u(\cdot, t)$ and $v(\cdot, t)$ on $\chi_{1}$ and $\chi_{2}$ will be a differentiable function of $t$, with derivative

$$
\left\langle\left(u_{t}, v_{t}\right),\left(\chi_{1}, \chi_{2}\right)\right\rangle=\left\langle\left(i\left[u_{x x}+a|u|^{2} u+b|v|^{2} u\right], \chi_{1}\right),\left(i\left[v_{x x}+b|u|^{2} v+c|v|^{2} v\right], \chi_{2}\right)\right\rangle
$$

which is a continuous function of $t$, where an application of (3.9) is used. Because $\phi_{\Omega} \in \mathcal{S}$, it follows that $Q$ is continuously differentiable with respect to $t$. 
We now proceed to show that the orbital stability result obtained in [17] for ground states can be improved in the sense explained above. Recall that the ground states are given by (2.4)-(1.8).

THEOREM 3.5. The ground-state solution $\left(e^{i \Omega t} A \phi_{\Omega}, e^{i \Omega t} B \phi_{\Omega}\right)$ is orbitally stable in the sense that for any $\epsilon>0$, there exists $\delta=\delta(\epsilon)>0$ such that if

$$
\inf _{\gamma_{1}, \gamma_{2}, y \in \mathbb{R}}\left\{\left\|u_{0}-e^{i \gamma_{1}} A \phi_{\Omega}(\cdot+y)\right\|_{H^{1}}+\left\|v_{0}-e^{i \gamma_{2}} B \phi_{\Omega}(\cdot+y)\right\|_{H^{1}}\right\}<\delta
$$

then there are $C^{1}$-mappings $\theta_{1}, \theta_{2}, \eta: \mathbb{R} \rightarrow \mathbb{R}$ for which the solution $(u(x, t), v(x, t))$ emanating from the initial data $(u(\cdot, 0), v(\cdot, 0))=\left(u_{0}, v_{0}\right)$ satisfies

$$
\left\|u(\cdot, t)-e^{i \theta_{1}(t)} A \phi_{\Omega}(\cdot-\eta(t))\right\|_{H^{1}}+\left\|v(\cdot, t)-e^{i \theta_{2}(t)} B \phi_{\Omega}(\cdot-\eta(t))\right\|_{H^{1}}<\epsilon
$$

for all $t \geq 0$. Moreover,

$$
\begin{aligned}
& \eta^{\prime}(t)=O(\epsilon), \\
& \theta_{i}^{\prime}(t)=\Omega+O(\epsilon)
\end{aligned}
$$

for $i=1,2$ as $\epsilon \rightarrow 0$, uniformly in $t$.

Proof. Proposition 3.4 guarantees that the function $Q$ is continuously differentiable with respect to $t$, hence we can differentiate $Q\left(u(\cdot, t), v(\cdot, t), \theta_{1}(t), \theta_{2}(t), \eta(t)\right)=$ $(0,0,0)$ with respect to the variable $t$ to obtain the following equations:

1) $\mathcal{R} e\left\{\int_{-\infty}^{\infty}\left(u_{t} A e^{-i \theta_{1}(t)} \phi_{\Omega}^{2} \phi_{\Omega}^{\prime}-i u A e^{-i \theta_{1}(t)} \theta_{1}^{\prime}(t) \phi_{\Omega}^{2} \phi_{\Omega}^{\prime}-\eta^{\prime}(t) u A e^{-i \theta_{1}(t)} \phi_{\Omega}^{2} \phi_{\Omega}^{\prime \prime}\right) d x\right.$

$$
\begin{aligned}
& +\int_{-\infty}^{\infty}\left(-2 u A e^{-i \theta_{1}(t)} \phi_{\Omega}\left(\phi_{\Omega}^{\prime}\right)^{2} \eta^{\prime}(t)-2 v B e^{-i \theta_{2}(t)} \phi_{\Omega}\left(\phi_{\Omega}^{\prime}\right)^{2} \eta^{\prime}(t)\right) d x \\
& \left.+\int_{-\infty}^{\infty}\left(v_{t} B e^{-i \theta_{2}(t)} \phi_{\Omega}^{2} \phi_{\Omega}^{\prime}-i v B e^{-i \theta_{2}(t)} \theta_{2}^{\prime}(t) \phi_{\Omega}^{2} \phi_{\Omega}^{\prime}-\eta^{\prime}(t) v B e^{-i \theta_{2}(t)} \phi_{\Omega}^{2} \phi_{\Omega}^{\prime \prime}\right) d x\right\}=0
\end{aligned}
$$

2) $\operatorname{Im}\left\{\int_{-\infty}^{\infty}\left(u_{t} A e^{-i \theta_{1}(t)} \phi_{\Omega}^{3}-i u A e^{-i \theta_{1}(t)} \theta_{1}^{\prime}(t) \phi_{\Omega}^{3}-3 \eta^{\prime}(t) u A e^{-i \theta_{1}(t)} \phi_{\Omega}^{2} \phi_{\Omega}^{\prime}\right) d x\right\}=0$;

3) $\mathcal{I} m\left\{\int_{-\infty}^{\infty}\left(v_{t} B e^{-i \theta_{2}(t)} \phi_{\Omega}^{3}-i v B e^{-i \theta_{2}(t)} \theta_{2}^{\prime}(t) \phi_{\Omega}^{3}-3 \eta^{\prime}(t) v B e^{-i \theta_{2}(t)} \phi_{\Omega}^{2} \phi_{\Omega}^{\prime}\right) d x\right\}=0$,

where $u=u(x+\eta(t), t), v=v(x+\eta(t), t)$ and $\phi_{\Omega}=\phi_{\Omega}(x)$. Define the functions $h$ and $k$ by

$$
\begin{aligned}
& h(x, t)=e^{-i \theta_{1}(t)} u(x+\eta(t), t)-A \phi_{\Omega}(x)=h_{1}+i h_{2}, \\
& k(x, t)=e^{-i \theta_{2}(t)} v(x+\eta(t), t)-B \phi_{\Omega}(x)=k_{1}+i k_{2} .
\end{aligned}
$$

Then

$$
e^{-i \theta_{1}(t)} u=A \phi_{\Omega}+h_{1}+i h_{2} \quad \text { and } \quad e^{-i \theta_{2}(t)} v=B \phi_{\Omega}+k_{1}+i k_{2} .
$$

The orbital stability result for $\left(e^{i \Omega t} A \phi_{\Omega}, e^{i \Omega t} B \phi_{\Omega}\right)$ obtained in [17] coupled with Lemma 3.2 imply the existence of $\delta>0$ such that if

$$
\inf _{\gamma_{1}, \gamma_{2}, y \in \mathbb{R}}\left\{\left\|u_{0}-A e^{i \gamma_{1}} \phi_{\Omega}(\cdot+y)\right\|_{H^{1}}+\left\|v_{0}-B e^{i \gamma_{2}} \phi_{\Omega}(\cdot+y)\right\|_{H^{1}}\right\}<\delta
$$


then the solution $(u(x, t), v(x, t))$ with $(u(\cdot, 0), v(\cdot, 0))=\left(u_{0}, v_{0}\right)$ satisfies

$$
\|h(x, t)\|_{H^{1}}+\|k(x, t)\|_{H^{1}}<\epsilon .
$$

Attention is now turned to the functions $\eta$ and $\theta_{i}$. Using the definitions of $h, k$ and the fact that $(u, v)$ must solve (3.9), the first equation in (3.10) can be expressed in terms of $h$ and $k$ rather than $u$ and $v$ as

$$
\begin{gathered}
\int_{-\infty}^{\infty}\left(A h_{2}^{\prime}\left(\phi_{\Omega}^{2} \phi_{\Omega}^{\prime}\right)^{\prime}-\left(a|u|^{2}+b|v|^{2}\right) h_{2} A \phi_{\Omega}^{2} \phi_{\Omega}^{\prime}+\theta_{1}^{\prime}(t) A h_{2} \phi_{\Omega}^{2} \phi_{\Omega}^{\prime}-\eta^{\prime}(t) A h_{1} \phi_{\Omega}^{2} \phi_{\Omega}^{\prime \prime}\right. \\
\left.-2 \eta^{\prime}(t) A^{2} \phi_{\Omega}^{2}\left(\phi_{\Omega}^{\prime}\right)^{2}-2 \eta^{\prime}(t) h_{1} A \phi_{\Omega}\left(\phi_{\Omega}^{\prime}\right)^{2}-\eta^{\prime}(t) A^{2} \phi_{\Omega}^{3} \phi_{\Omega}^{\prime \prime}\right) d x \\
+\int_{-\infty}^{\infty}\left(B k_{2}^{\prime}\left(\phi_{\Omega}^{2} \phi_{\Omega}^{\prime}\right)^{\prime}-\left(b|u|^{2}+c|v|^{2}\right) k_{2} B \phi_{\Omega}^{2} \phi_{\Omega}^{\prime}+\theta_{2}^{\prime}(t) B k_{2} \phi_{\Omega}^{2} \phi_{\Omega}^{\prime}-\eta^{\prime}(t) B k_{1} \phi_{\Omega}^{2} \phi_{\Omega}^{\prime \prime}\right. \\
\left.-2 \eta^{\prime}(t) B^{2} \phi_{\Omega}^{2}\left(\phi_{\Omega}^{\prime}\right)^{2}-2 \eta^{\prime}(t) k_{1} B \phi_{\Omega}\left(\phi_{\Omega}^{\prime}\right)^{2}-\eta^{\prime}(t) B^{2} \phi_{\Omega}^{3} \phi_{\Omega}^{\prime \prime}\right) d x=0 .
\end{gathered}
$$

Because of (3.12) and the fact that $\|u(\cdot, t)\|_{L^{2}}+\|v(\cdot, t)\|_{L^{2}} \leq C$ for some constant $C>0$ independent of $t$, one can readily justify that

$$
\eta^{\prime}(t)=O(\epsilon)+O(\epsilon)\left(\theta_{1}^{\prime}(t)+\theta_{2}^{\prime}(t)\right)
$$

as $\epsilon \rightarrow 0$, uniformly in $t$. Similarly, the second equation in (3.10) can be rewritten as

$$
\begin{gathered}
\int_{-\infty}^{\infty}\left(A^{2} \phi_{\Omega}^{\prime \prime} \phi_{\Omega}^{3}+A h_{1}\left(\phi_{\Omega}^{3}\right)^{\prime \prime}+A^{2} \phi_{\Omega}^{4}\left(a|u|^{2}+b|v|^{2}\right)+\left(a|u|^{2}+b|v|^{2}\right) A h_{1} \phi_{\Omega}^{3}\right. \\
\left.-\theta_{1}^{\prime}(t) A^{2} \phi_{\Omega}^{4}-\theta_{1}^{\prime}(t) A h_{1} \phi_{\Omega}^{3}-3 \eta^{\prime}(t) A h_{2} \phi_{\Omega}^{2} \phi_{\Omega}^{\prime}\right) d x=0
\end{gathered}
$$

from which one can deduce, using (2.5)-(3.12), that

$$
\theta_{1}^{\prime}(t) \int_{-\infty}^{\infty} A^{2} \phi_{\Omega}^{4}=\int_{-\infty}^{\infty} A^{2} \phi_{\Omega}^{3} \phi_{\Omega}^{\prime \prime}+A^{2} \phi_{\Omega}^{4}\left(a|u|^{2}+b|v|^{2}\right)+O(\epsilon)+O(\epsilon) \eta^{\prime}(t)
$$

as $\epsilon \rightarrow 0$, uniformly in $t$. It is straightforward to see that

$$
\int_{-\infty}^{\infty} A^{2} \phi_{\Omega}^{4}\left(a|u|^{2}+b|v|^{2}\right)=\int_{-\infty}^{\infty} A^{2} \phi_{\Omega}^{4}\left(a\left|A \phi_{\Omega}\right|^{2}+b\left|B \phi_{\Omega}\right|^{2}\right)+O(\epsilon)
$$

as $\epsilon \rightarrow 0$, uniformly in $t$. It is then concluded using (3.14), (3.15), and the first equation in $(2.5)$ that

$$
\theta_{1}^{\prime}(t)=\Omega+O(\epsilon)+O(\epsilon) \eta^{\prime}(t)
$$

as $\epsilon \rightarrow 0$, uniformly in $t$. Likewise, the last equation in (3.10) can be written in terms of $h$ and $k$ as

$$
\begin{gathered}
\int_{-\infty}^{\infty}\left(B^{2} \phi_{\Omega}^{\prime \prime} \phi_{\Omega}^{3}+B k_{1}\left(\phi_{\Omega}^{3}\right)^{\prime \prime}+B^{2} \phi_{\Omega}^{4}\left(b|u|^{2}+c|v|^{2}\right)+\left(b|u|^{2}+c|v|^{2}\right) B k_{1} \phi_{\Omega}^{3}\right. \\
\left.-\theta_{2}^{\prime}(t) B^{2} \phi_{\Omega}^{4}-\theta_{2}^{\prime}(t) B k_{1} \phi_{\Omega}^{3}-3 \eta^{\prime}(t) B k_{2} \phi_{\Omega}^{2} \phi_{\Omega}^{\prime}\right) d x=0
\end{gathered}
$$


which gives

$$
\theta_{2}^{\prime}(t)=\Omega+O(\epsilon)+O(\epsilon) \eta^{\prime}(t)
$$

as $\epsilon \rightarrow 0$, uniformly in $t$. We can now deduce from (3.13), (3.16) and (3.17) that

$$
\begin{aligned}
& \eta^{\prime}(t)=O(\epsilon), \\
& \theta_{i}^{\prime}(t)=\Omega+O(\epsilon)
\end{aligned}
$$

for $i=1,2$ as $\epsilon \rightarrow 0$, uniformly in $t$.

\section{Result for solitary-wave solutions}

The result obtained in Section 3 is now broadened to include solitary-wave solutions and improved by providing a more detailed view of the connection between the functions $\eta$ and $\theta_{i}$. Using the same approach employed in [8], a relation between ground states and solitary-wave solutions is first exhibited as follows. For $\theta \in \mathbb{R}$, define the operator $T_{\theta}: H_{\mathbb{C}}^{1}(\mathbb{R}) \rightarrow H_{\mathbb{C}}^{1}(\mathbb{R})$ by

$$
\left(T_{\theta} u\right)(x)=\exp \left(\frac{i \theta x}{2}\right) u(x)
$$

for $u \in H_{\mathbb{C}}^{1}(\mathbb{R})$. Then for any $\theta \in \mathbb{R}$,

$$
(1+|\theta|)^{-1}\|u\|_{H^{1}} \leq\left\|T_{\theta} u\right\|_{H^{1}} \leq(1+|\theta|)\|u\|_{H^{1}} .
$$

For any pair $(\omega, \theta) \in \mathbb{R} \times \mathbb{R}$ such that $\Omega=\omega-\frac{1}{4} \theta^{2}>0$, let

$$
\varphi_{\omega}=T_{\theta} \phi_{\Omega} .
$$

A straightforward calculation reveals the following lemma whose proof is omitted.

Lemma 4.1. If $\left(e^{i \Omega t} A \phi_{\Omega}, e^{i \Omega t} B \phi_{\Omega}\right)$ is a ground state solution of (1.1), then $\left(e^{i \omega t} A \varphi_{\omega}, e^{i \omega t} B \varphi_{\omega}\right)$ is a solitary-wave solution of (1.1).

The stability theory for solitary-wave solutions is as follows.

THEOREM 4.2. The solitary-wave solutions $\left(e^{i \omega t} A \varphi_{\omega}, e^{i \omega t} B \varphi_{\omega}\right)$ are orbitally stable in the sense that for any $\epsilon>0$ given, there exists $\delta=\delta(\epsilon)>0$ such that if

$$
\inf _{\gamma_{1}, \gamma_{2}, y \in \mathbb{R}}\left\{\left\|u_{0}-e^{i \gamma_{1}} A \varphi_{\omega}(\cdot+y)\right\|_{H^{1}}+\left\|v_{0}-e^{i \gamma_{2}} B \varphi_{\omega}(\cdot+y)\right\|_{H^{1}}\right\} \leq \delta
$$

then there are $C^{1}$-mappings $p_{1}, p_{2}, q: \mathbb{R} \rightarrow \mathbb{R}$ for which the solution $(u, v)$ of (1.1) emanating from the initial data $\left(u_{0}, v_{0}\right)$ satisfies

$$
\left\|u(\cdot, t)-e^{i p_{1}(t)} A \varphi_{\omega}(\cdot-q(t))\right\|_{H^{1}}+\left\|v(\cdot, t)-e^{i p_{2}(t)} B \varphi_{\omega}(\cdot-q(t))\right\|_{H^{1}} \leq \epsilon
$$

for all $t \geq 0$. Moreover, $p_{1}, p_{2}$, and $q$ are close to $\omega$ and $\theta$ in the sense that

$$
\begin{aligned}
& p_{1}^{\prime}(t)=\omega+O(\epsilon), \\
& p_{2}^{\prime}(t)=\omega+O(\epsilon), \\
& q^{\prime}(t)=\theta+O(\epsilon)
\end{aligned}
$$

as $\epsilon \rightarrow 0$, uniformly in $t$. 
We need the following calculations before we present the proof of Theorem 4.2. For $(u, v)$ a solution of the initial-value problem for (1.1) with initial data $\left(u_{0}, v_{0}\right)$, define

$$
\begin{aligned}
& f(x, t)=e^{i\left(\frac{1}{2} \theta x-\frac{1}{4} \theta^{2} t\right)} u(x-\theta t, t) ; \\
& g(x, t)=e^{i\left(\frac{1}{2} \theta x-\frac{1}{4} \theta^{2} t\right)} v(x-\theta t, t) .
\end{aligned}
$$

The proof of the following Lemma is straightforward, hence omitted.

LEMma 4.3. Let $(u, v)$ be a solution of the initial-value problem for (1.1) with initial data $\left(u_{0}, v_{0}\right)$. Then the pair $(f, g)$ defined as in (4.3) is a solution of the initial-value problem

$$
\left\{\begin{array}{c}
i f_{t}+f_{x x}+\left(a|f|^{2}+b|g|^{2}\right) f=0, \\
i g_{t}+g_{x x}+\left(b|f|^{2}+c|g|^{2}\right) g=0
\end{array}\right.
$$

with initial data $(f(x, 0), g(x, 0))=\left(f_{0}, g_{0}\right)=\left(T_{\theta} u_{0}, T_{\theta} v_{0}\right)$.

Consider initial data $\left(f_{0}, g_{0}\right)$ which lies close to a solitary wave $\left(e^{i \omega t} A \varphi_{\omega}, e^{i \omega t} B \varphi_{\omega}\right)$ in $X$ and define

$$
\left(u_{0}, v_{0}\right)=\left(T_{-\theta} f_{0}, T_{-\theta} g_{0}\right) .
$$

Let $(f, g)$ and $(u, v)$ be the solutions of (1.1) with initial data $\left(f_{0}, g_{0}\right)$ and $\left(u_{0}, v_{0}\right)$ respectively. Notice that from (4.1),

$$
\begin{aligned}
& \left\|u_{0}-e^{i \gamma_{1}} A \phi_{\Omega}\right\|_{H^{1}}+\left\|v_{0}-e^{i \gamma_{2}} B \phi_{\Omega}\right\|_{H^{1}} \\
= & \left\|T_{-\theta} f_{0}-e^{i \gamma_{1}} A T_{-\theta} \varphi_{\omega}\right\|_{H^{1}}+\left\|T_{-\theta} g_{0}-e^{i \gamma_{1}} B T_{-\theta} \varphi_{\omega}\right\|_{H^{1}} \\
\leq & (1+|\theta|)\left\|f_{0}-e^{i \gamma_{1}} A \varphi_{\omega}\right\|_{H^{1}}+(1+|\theta|)\left\|g_{0}-e^{i \gamma_{2}} B \varphi_{\omega}\right\|_{H^{1}} .
\end{aligned}
$$

Proof. (of Theorem 4.2.) Let $\epsilon>0$ be fixed, let $\tilde{\epsilon}=\epsilon /(1+|\theta|)$, and let $\delta$ be such that the stability result established in Theorem 3.5 holds relative to $\tilde{\epsilon}$ for the ground state $\left(e^{i \Omega t} A \phi_{\Omega}(x, t), e^{i \Omega t} B \phi_{\Omega}(x, t)\right)$, that is,

$$
\left\|u(\cdot, t)-e^{i \theta_{1}(t)} A \phi_{\Omega}(\cdot-\eta(t))\right\|_{H^{1}}+\left\|v(\cdot, t)-e^{i \theta_{2}(t)} B \phi_{\Omega}(\cdot-\eta(t))\right\|_{H^{1}}<\tilde{\epsilon}
$$

for all $t \geq 0$, provided

$$
\inf _{\gamma_{1}, \gamma_{2}, y \in \mathbb{R}}\left\{\left\|u_{0}-e^{i \gamma_{1}} A \phi_{\Omega}(\cdot+y)\right\|_{H^{1}}+\left\|v_{0}-e^{i \gamma_{2}} B \phi_{\Omega}(\cdot+y)\right\|_{H^{1}}\right\}<\delta .
$$

But then according to (4.4),

$$
\inf _{\gamma_{1}, \gamma_{2}, y \in \mathbb{R}}\left\{\left\|u_{0}-e^{i \gamma_{1}} A \phi_{\Omega}(\cdot+y)\right\|_{H^{1}}+\left\|v_{0}-e^{i \gamma_{2}} B \phi_{\Omega}(\cdot+y)\right\|_{H^{1}}\right\} \leq \delta
$$

provided $\left\|f_{0}-e^{i \gamma_{1}} A \varphi_{\omega}\right\|_{H^{1}}+\left\|g_{0}-e^{i \gamma_{2}} B \varphi_{\omega}\right\|_{H^{1}} \leq \delta /(1+\theta)$. Now, because of Lemma 4.3 , it follows that

$$
\begin{aligned}
& u(x, t)=e^{-i\left(\frac{1}{2} \theta x+\frac{1}{4} \theta^{2} t\right)} f(x+\theta t, t) ; \\
& v(x, t)=e^{-i\left(\frac{1}{2} \theta x+\frac{1}{4} \theta^{2} t\right)} g(x+\theta t, t) .
\end{aligned}
$$


Hence (4.5) can be rewritten as

$$
\begin{aligned}
& \left\|e^{-\frac{i}{2} \theta x}\left\{e^{-\frac{i}{4} \theta^{2} t} f(\cdot+\theta t, t)-e^{i\left[\theta_{1}(t)+\frac{1}{2} \theta \eta(t)\right]} A \varphi_{\omega}(\cdot-\eta(t))\right\}\right\|_{H^{1}} \\
& \left\|e^{-\frac{i}{2} \theta x}\left\{e^{-\frac{i}{4} \theta^{2} t} g(\cdot+\theta, t)-e^{i\left[\theta_{2}(t)+\frac{1}{2} \theta \eta(t)\right]} B \varphi_{\omega}(\cdot-\eta(t))\right\}\right\|_{H^{1}} \leq \tilde{\epsilon}
\end{aligned}
$$

for all $t \geq 0$. Using the above inequality and (4.1), it is concluded that

$$
\begin{aligned}
& \left\|f(\cdot, t)-e^{i\left(\theta_{1}(t)+\frac{1}{4} \theta^{2} t+\frac{1}{2} \theta \eta(t)\right)} A \varphi_{\omega}(\cdot-\eta(t)-\theta t)\right\|_{H^{1}} \\
& +\left\|g(\cdot, t)-e^{i\left(\theta_{2}(t)+\frac{1}{4} \theta^{2} t+\frac{1}{2} \theta \eta(t)\right)} B \varphi_{\omega}(\cdot-\eta(t)-\theta t)\right\|_{H^{1}} \leq(1+|\theta|) \tilde{\epsilon}=\epsilon
\end{aligned}
$$

for all $t \geq 0$. Thus $\left(e^{i \omega t} A \varphi_{\omega}, e^{i \omega t} B \varphi_{\omega}\right)$ is seen to be orbitally stable in the sense specified in the statement of the Theorem if one chooses

$$
\begin{aligned}
& p_{1}(t)=\theta_{1}(t)+\frac{1}{4} \theta^{2} t+\frac{1}{2} \theta \eta(t) ; \\
& p_{2}(t)=\theta_{2}(t)+\frac{1}{4} \theta^{2} t+\frac{1}{2} \theta \eta(t) ; \\
& q(t)=\eta(t)+\theta t .
\end{aligned}
$$

This completes the proof of Theorem 4.2 .

\section{Conclusion}

The solitary-wave solutions of (1.1) have been shown to be orbitally stable in $X$. Moreover, it was established that the solution emanating from a perturbed solitary wave travels at nearly the same speed and phase shifts as the unperturbed solitary wave.

There are, however, some very interesting questions that remain open. First, are these waves stable to rougher perturbations? For example, it has been shown in [16] that for the Korteweg-de Vries equation, the solitary waves are stable even in $L^{2}(\mathbb{R})$. Second, are those solitary waves also stable in smaller spaces such as $H_{\mathbb{C}}^{s}(\mathbb{R}) \times H_{\mathbb{C}}^{s}(\mathbb{R})$ for $s>1$ ? Again, the solitary waves for the nonlinear Schrödinger equation (1.2) and Korteweg-de Vries equation, for example, are known to be stable in $H^{k}(\mathbb{R})$ for $k=2,3,4, \ldots$ (see $[7])$.

Acknowledgement. The author would like to thank the referees for their valuable comments, suggestions and for pointing out some related references.

\section{REFERENCES}

[1] A. Ambrosetti and E. Colorado, Bound and ground states of coupled nonlinear Schrödinger equations, C.R. Math. Acad. Sci. Paris, 342, 453-458, 2006.

[2] A. Ambrosetti and E. Colorado, Standing waves of some coupled nonlinear Schrödinger equations, J. London Math. Soc., 75, 67-82, 2007.

[3] T. Bartsch, N. Dancer, and Z.Q. Wang, A Liouville theorem, a-priori bounds, and bifurcating branches of positive solutions for a nonlinear elliptic system, Cal. of Var. and PDEs, no. 3-4, 345-361, 2010.

[4] T. Bartsch and Z.Q. Wang, Note on ground states of nonlinear Schrödinger systems, J. Part. Diff. Eqs., 19, 200-207, 2006.

[5] T. Bartsch, Z.-Q. Wang, and J. Wei, Bound states for a coupled Schrödinger system, J. Fixed Point Theory Appl., 2, 353-367, 2007. 
[6] D.J. Benney and A.C. Newell, The propagation of nonlinear wave envelopes, J. Math. Phys., 46, 133-139, 1967.

[7] J.L. Bona, Y. Liu, and N.V. Nguyen, Stability of solitary waves in higher-order Sobolev spaces, Commun. Math Sci., 2(1), 35-52, 2004.

[8] J.L. Bona and A. Soyeur, On the stability of solitary-wave solutions of model equations for long waves, Jour. Nonlin. Sci., 4, 449-470, 1994.

[9] T. Cazenave, An Introduction to Nonlinear Schrödinger Equations, Textos de Métodos Matemáticos, Instituto de Matemática-UFRJ, Rio de Janeiro, 22, 1989.

[10] R. Cipolatti and W. Zumpichiatti, Orbitally stable standing waves for a system of coupled nonlinear Schrödinger equations, Nonlinear Anal., 42, 445-461, 2000.

[11] E.N. Dancer, J. Wei, and T. Weth, A priori bounds versus multiple existence of positive solutions for a nonlinear Schrödinger system, Ann. Inst. H. Poincare Anal. Non Linearaire, 3(27), 953-969, 2010.

[12] A. Hasegawa and F. Tappert, Transmission of stationary nonlinear optical pulses in dispersive dielectric fibers I. Anomalous dispersion, Appl. Phys. Lett., 23, 142, 1973.

[13] A. Hasegawa and F. Tappert, Transmission of stationary nonlinear optical pulses in dispersive dielectric fibers II. Normal dispersion, Appl. Phys. Lett., 23, 171, 1973.

[14] T.C. Lin and J. Wei, Ground state of $N$ coupled nonlinear Schrödinger equations in $R^{n}, n \leq 3$, Commun. Math. Phys., 255, 629-653, 2005.

[15] Z. Liu and Z.Q. Wang, Multiple bound states of nonlinear Schrödinger systems, Commun. Math. Phys., 282, 721-731, 2008.

[16] F. Merle and L. Vega, $L^{2}$ stability of solitons for $K d V$ equations, Int. Math. Res. Notes, 13, 735-753, 2003.

[17] N.V. Nguyen and Z.Q. Wang, Orbital stability of solitary waves for a nonlinear Schrödinger system, preprint.

[18] M. Ohta, Stability of solitary waves for coupled nonlinear Schrödinger equations, Nonlinear Anal.Theory, Methods \& Appl., 26(5), 933-939, 1996.

[19] B. Sirakov, Least energy solitary waves for a system of nonlinear Schrödinger equations in $\mathbb{R}^{n}$, Commun. Math. Phys., 271, 199-221, 2007.

[20] X. Song, Stability and instability of standing waves to a system of Schrödinger equations with combined power-type nonlinearities, J. Math. Anal. Appl., 366, 345-359, 2010.

[21] X. Song, Sharp thresholds of global existence and blowup for a system of Schrödinger equations with combined power-type nonlinearities, J. Math. Phys., 51, 033509, 2010.

[22] J. Wei and W. Yao, Uniqueness of positive solutions to some coupled nonlinear Schrödinger equations, preprint.

[23] M. Weinstein, Lyapunov stability of ground states of nonlinear dispersive evolution equations, Commun. Pure Appl. Math., 39, 51-68, 1986.

[24] V.E. Zakharov, Stability of periodic waves of finite amplitude on the surface of a deep fluid, Sov. Phys. Jour. Appl. Mech. Tech. Phys., 4, 190-194, 1968.

[25] V.E. Zakharov, Collapse of Langmuir waves, Sov. Phys. JETP, 35, 908-914, 1972. 